Supporting Information for:

\title{
PET-RAFT and SAXS: High Throughput Tools To Study Compactness and Flexibility of Single Chain Polymer Nanoparticles
}

\author{
Rahul Upadhya ${ }^{1}$, N. Sanjeeva Murthy ${ }^{2}$, Cody L. Hoop, ${ }^{3}$ Shashank Kosuri ${ }^{1}$, Vikas Nanda, ${ }^{4}$ \\ Joachim Kohn, ${ }^{2}$ Jean Baum ${ }^{3}$, Adam J. Gormley*1
}

${ }^{1}$ Department of Biomedical Engineering, Rutgers, The State University of New Jersey, Piscataway, NJ 08854, USA

${ }^{2}$ New Jersey Center for Biomaterials, Rutgers, The State University of New Jersey, Piscataway, NJ 08854, USA

${ }^{3}$ Department of Chemistry and Chemical Biology, Rutgers, The State University of New Jersey, Piscataway, NJ 08854, USA

${ }^{4}$ Center for Advanced Biotechnology and Medicine and the Department of Biochemistry and Molecular Biology,

Robert Wood Johnson Medical School, Rutgers, The State University of New Jersey, Piscataway, NJ 08854, USA

Corresponding Author E-mail: Adam.Gormley@ rutgers.edu

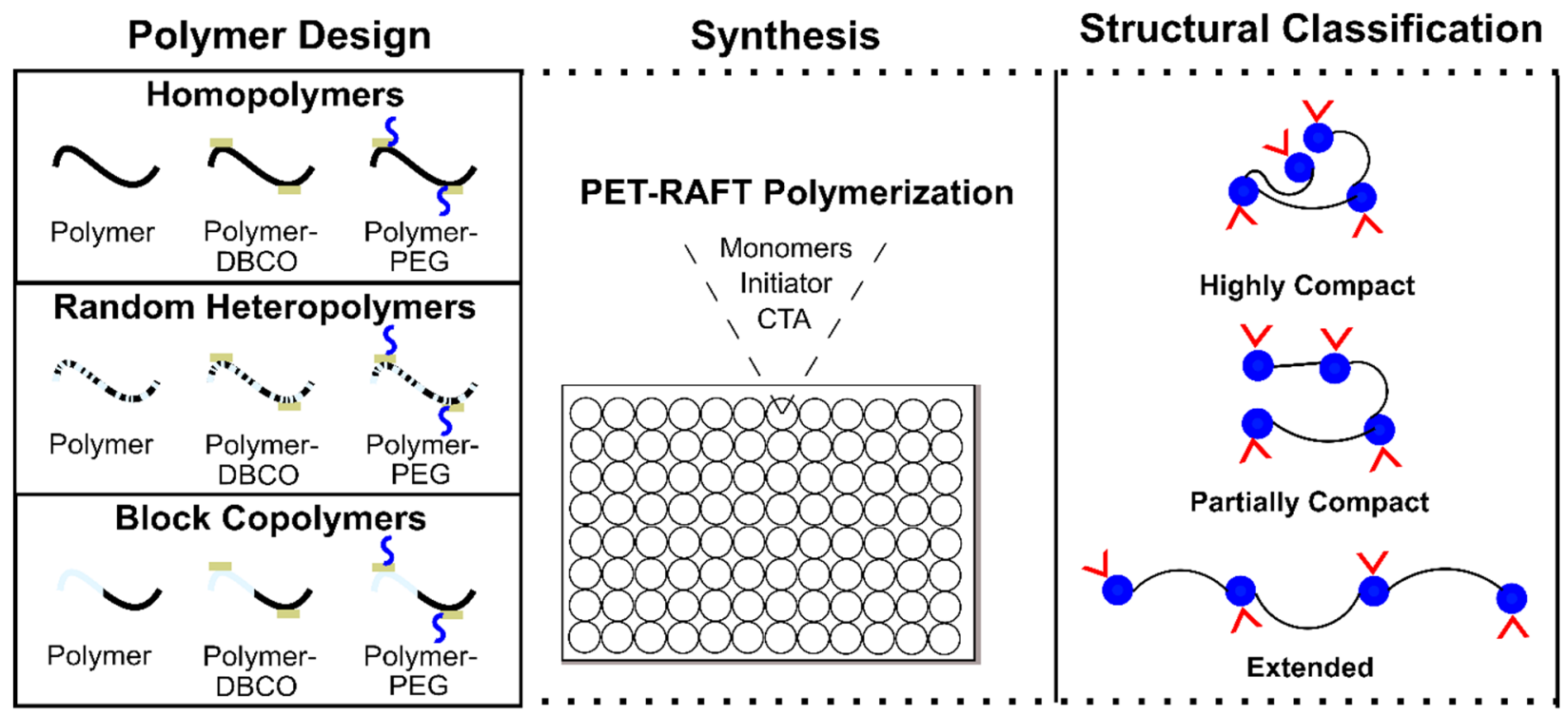




\section{S1. Supplemental Methods}

\section{S1.1: Definition of Homopolymers, Random Heteropolymers, and Block Copolymers}

The general definition of homopolymers, random heteropolymers, and block copolymers is presented in Table S1. Each monomer column contains the number of units designed for the polymer chain (n) with the mol\% (\%). Included are definitions for all polymer backbones (labeled: homopolymer, $\mathrm{RH} / \mathrm{BC}(1: 1), \mathrm{RH} / \mathrm{BC}(1: 1: 1)$, and $\mathrm{RH} / \mathrm{BC}$ (9:1), where $\mathrm{RH}=$ random heteropolymer, $\mathrm{BC}=$ block copolymer, and the included ratios are the feed ratios of monomer 1 : monomer 2).

Table S1. Summary of definitions for all polymer sub-types: homopolymers, random heteropolymers (RH), block copolymers (BC), and functionalized polymers.

\begin{tabular}{|c|c|c|c|c|c|c|c|}
\hline Polymer Sub-Type & $\begin{array}{c}\text { Monomer } 1 \\
n / \%\end{array}$ & $\begin{array}{c}\text { Monomer } 2 \\
\text { n / \% }\end{array}$ & $\begin{array}{c}\text { Monomer } 3 \\
\mathrm{n} / \%\end{array}$ & $\begin{array}{l}\text { NHS } \\
\mathrm{n} / \%\end{array}$ & $\begin{array}{c}\text { DBCO- } \mathrm{NH}_{2} \\
\mathrm{n} / \%\end{array}$ & $\begin{array}{l}\text { PEG-N }{ }_{3} \\
\mathrm{n} / \%\end{array}$ & $\begin{array}{c}\text { SI } \\
\text { Table }\end{array}$ \\
\hline Homopolymer & $400 / 100 \%$ & - & - & - & - & - & Table S6 \\
\hline $\begin{array}{c}\text { Homopolymer } \\
\text { (PEG-Functionalized) }\end{array}$ & $360 / 75 \%$ & - & - & $40 / 8.33 \%$ & $40 / 8.33 \%$ & $40 / 8.33 \%$ & Table S6 \\
\hline $\mathrm{RH} / \mathrm{BC}(1: 1)$ & $200 / 50 \%$ & $200 / 50 \%$ & - & - & - & - & Table S7 \\
\hline $\begin{array}{c}\mathrm{RH} / \mathrm{BC}(1: 1) \\
\text { (PEG-Functionalized) }\end{array}$ & $180 / 37.5 \%$ & $180 / 37.5 \%$ & - & $40 / 8.33 \%$ & $40 / 8.33 \%$ & $40 / 8.33 \%$ & Table S7 \\
\hline $\mathrm{RH} / \mathrm{BC}(1: 1: 1)$ & $133 / 33.3 \%$ & $133 / 33.3 \%$ & $133 / 33.3 \%$ & - & - & - & Table S7 \\
\hline $\begin{array}{c}\text { RH/BC (1:1:1) } \\
\text { (PEG-Functionalized) }\end{array}$ & $120 / 25 \%$ & $120 / 25 \%$ & $120 / 25 \%$ & $40 / 8.33 \%$ & $40 / 8.33 \%$ & $40 / 8.33 \%$ & Table S7 \\
\hline RH/BC (9:1) & $360 / 90 \%$ & $40 / 10 \%$ & - & - & - & - & Tables S8-S11 \\
\hline $\begin{array}{c}\mathrm{RH} / \mathrm{BC}(9: 1) \\
\text { (PEG-Functionalized) }\end{array}$ & $324 / 67.5 \%$ & $36 / 7.5 \%$ & - & $40 / 8.33 \%$ & $40 / 8.33 \%$ & $40 / 8.33 \%$ & Tables S8-S11 \\
\hline
\end{tabular}

\section{S.1.2: $\log P$ Calculations}

$\log P$ was calculated for each of the groups displayed in Table $\mathrm{S} 1$ by taking the weighted average of components as follows:

$$
\begin{gathered}
\log P=\left(\log P_{m o n 1} * x_{m o n 1}\right)+\left(\log P_{m o n 2} * x_{m o n 2}\right)+\left(\log P_{m o n 3} * x_{m o n 3}\right)+\left(\log P_{N H S} * x_{N H S}\right) \\
+\left(\log P_{D B C O} * x_{D B C O}\right)+\left(\left(\log P_{P E G} * x_{P E G}\right)\right.
\end{gathered}
$$

where $\mathrm{x}$ refers to the mol\% of the individual monomers, NHS acrylic acid, DBCO-NH $\mathrm{N}_{2}$, or PEG$\mathrm{N}_{3}$.

This can also be re-expressed as:

$$
\log P=\sum_{i}\left(\log P_{i} * x_{i}\right)
$$

where $\mathrm{i}=1,2,3$, NHS, DBCO-NH 2 , and PEG-N $\mathrm{N}_{3}$. 


\section{S2. Additional Information}

HEA 2-HPMA Neutral

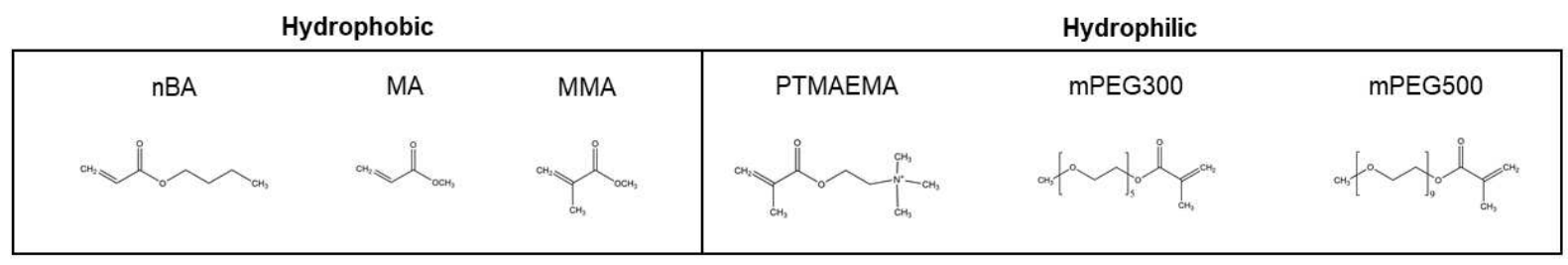

\begin{tabular}{|c|c|}
\hline \multicolumn{2}{c}{ Conjugation Tools } \\
\hline $\begin{array}{c}\mathrm{NHS} \\
\text { acrylic acid }\end{array}$ \\
\hline
\end{tabular}

Figure S1. Chemical structures of neutral monomers (HEA, NAM, 2-HPMA, HEMA, mPEG acrylate, and NIPAM), hydrophobic monomers (nBA, MA, and MMA), hydrophilic monomers (PTMAEMA, mPEG300, and mPEG500), and conjugation tools (NHS acrylic acid, DBCO-NH 2 , and PEG- $\mathrm{N}_{3}$ ).

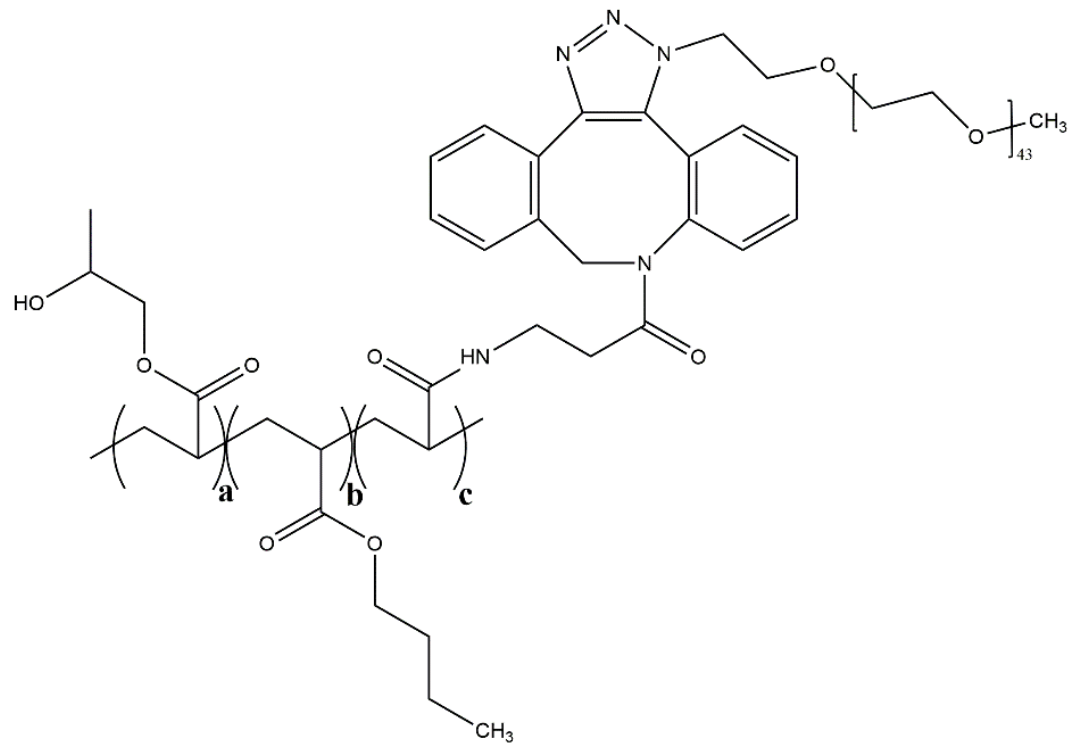

Figure S2. Illustrating the structure of a PEG-functionalized random heteropolymer (RH 2-HPMA-

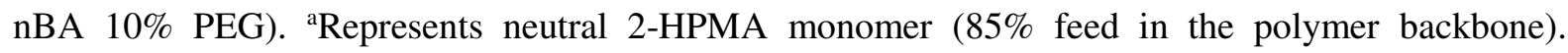
${ }^{b}$ Represents hydrophobic nBA monomer (5\% feed in the polymer backbone). ${ }^{\mathrm{c}}$ Represents the portion of the polymer chain at which NHS acrylic acid was originally polymerized (10\% feed). 


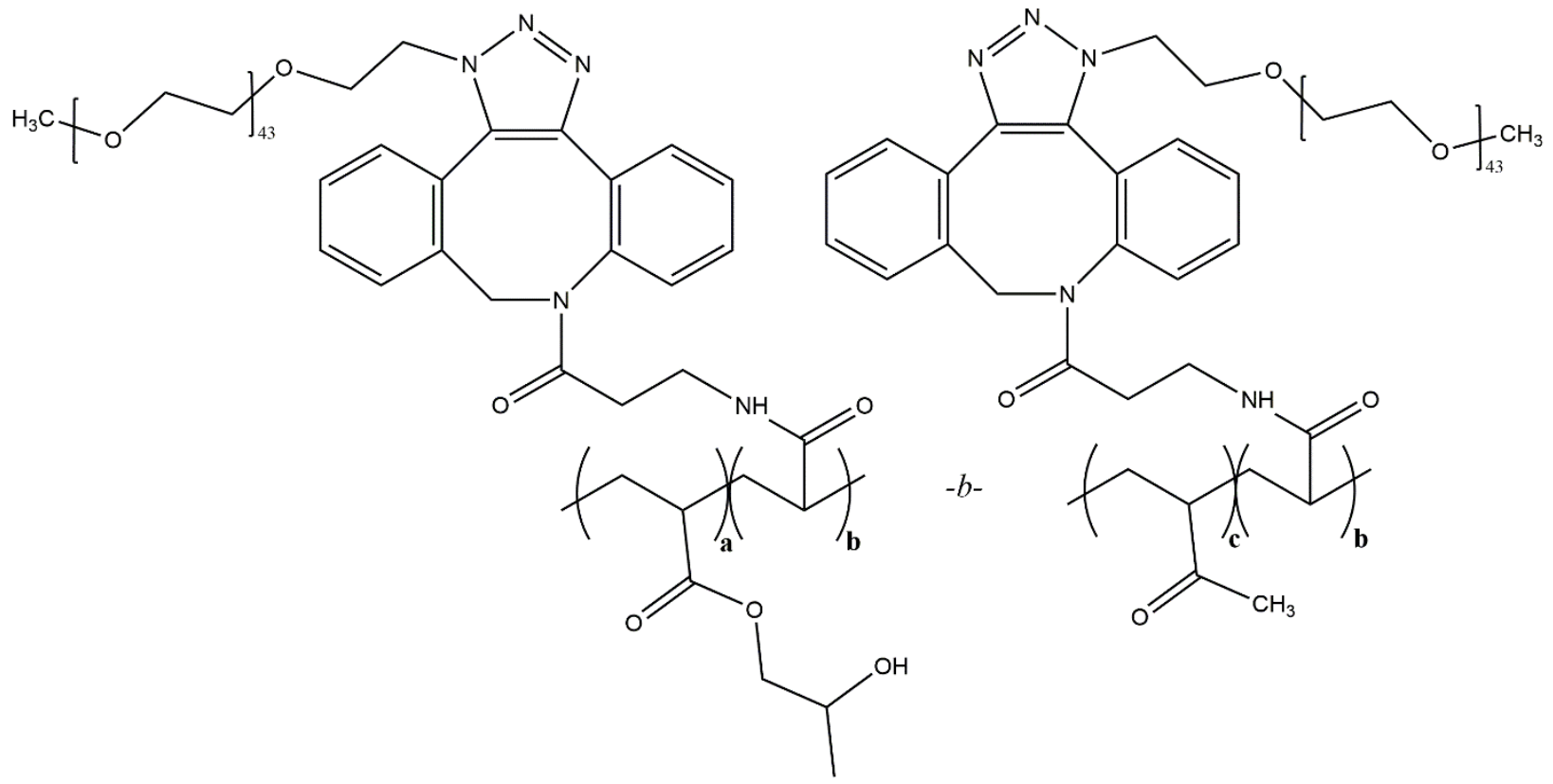

Figure S3. Illustrating the structure of a PEG-functionalized block copolymer (BC 2-HPMA-MA 10\% PEG). ${ }^{a}$ Represents neutral 2-HPMA monomer ( $85 \%$ feed in the polymer backbone). ${ }^{\text {b Represents the }}$ portion of the polymer chain at which NHS acrylic acid was originally polymerized ( $10 \%$ feed in the polymer backbone). ${ }^{c}$ Represents the hydrophobic nBA monomer (5\% feed in the polymer backbone). Note that this design was selected in order for functionalization to be evenly dispersed.

Table S2. $\log$ P values determined for each compound.

\begin{tabular}{cc} 
Sample & logP \\
HEA & 0.57 \\
NAM & -0.13 \\
DMA & 0.21 \\
NIPAM & 0.72 \\
2-HPMA & 1.26 \\
HEMA & 0.85 \\
mPEG acrylate & 0.33 \\
nBA & 2.52 \\
MA & 0.49 \\
MMA & 1.59 \\
mPEG300 & 0.77 \\
mPEG500 & 0.11 \\
PTMAEMA & -6.90 \\
NHS acrylate & 0.65 \\
DBCO-NH & \\
PEG-N & 2.14 \\
\hline
\end{tabular}




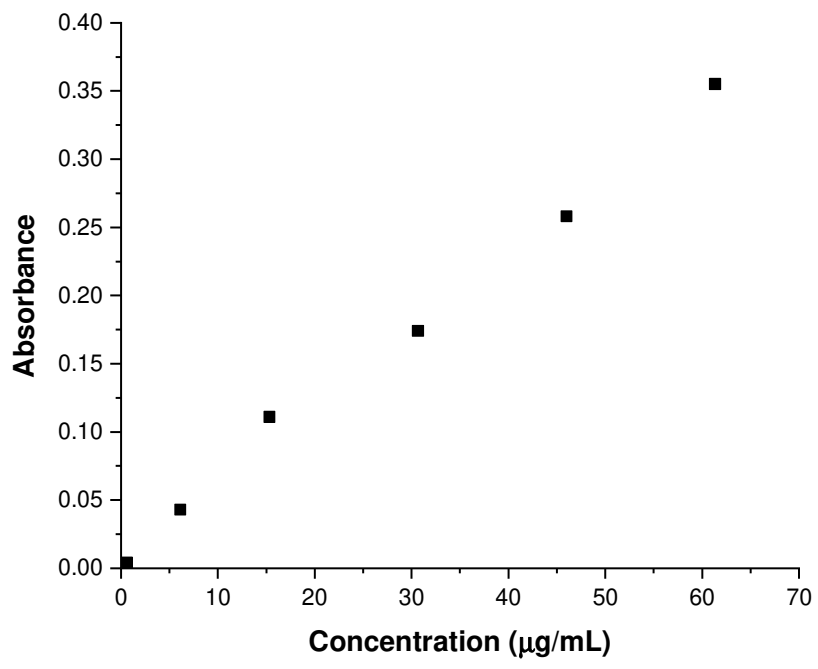

Figure S4. Standard curve for $\mathrm{DBCO}-\mathrm{NH}_{2}$ that was used to determine all concentrations postpurification for functionalization at a wavelength of $310 \mathrm{~nm}$.

Table S3. DBCO incorporation percentages of all homopolymers. A background subtraction of polymer-NHS was done from the previous step to obtain only the concentration of DBCO present.

$\begin{array}{cc}\text { Polymer } & \text { DBCO Incorporation \% } \\ \text { HEA } & 5.00 \\ \text { NAM } & 4.07 \\ \text { NIPAM } & 3.84 \\ \text { DMA } & 5.27 \\ \text { 2-HPMA } & 1.28 \\ \text { HEMA } & 1.66 \\ \text { mPEG acrylate } & 2.59\end{array}$


Table S4. DBCO incorporation percentages of all random heteropolymers. A background subtraction of polymer-NHS was done from the previous step to obtain only the concentration of DBCO present.

\begin{tabular}{|c|c|}
\hline Polymer & DBCO Incorporation \% \\
\hline RH HEA-NIPAM & 12.1 \\
\hline RH HEA-2-HPMA & 4.07 \\
\hline RH HEA-HEMA & 4.60 \\
\hline RH HEA-NAM & 13.4 \\
\hline RH HEA-NAM-HEA & 13.8 \\
\hline RH HEA-mPEG300 & 10.5 \\
\hline RH HEA-mPEG500 & 11.2 \\
\hline RH HEA-PTMAEMA & 4.71 \\
\hline RH HEA-nBA & 10.8 \\
\hline RH HEA-MA & 10.8 \\
\hline RH HEA-MMA & 12.4 \\
\hline RH NIPAM-2-HPMA & 5.50 \\
\hline RH NIPAM-PTMAEMA & 5.47 \\
\hline RH NIPAM-nBA & 8.58 \\
\hline RH NIPAM-MA & 11.2 \\
\hline RH NIPAM-MMA & 9.57 \\
\hline RH 2-HPMA-nBA & 9.54 \\
\hline RH 2-HPMA-MA & 3.04 \\
\hline RH HEA-MMA & 12.4 \\
\hline RH 2-HPMA-MMA & 10.4 \\
\hline RH HEMA-nBA & 6.57 \\
\hline RH HEMA-MA & 3.58 \\
\hline RH HEMA-MMA & 9.31 \\
\hline
\end{tabular}


Table S5. DBCO incorporation percentages of all block copolymers. A background subtraction of polymer-NHS was done from the previous step to obtain only the concentration of DBCO present.

\begin{tabular}{cc} 
Polymer & DBCO Incorporation \% \\
BC HEA-NIPAM & 11.1 \\
BC HEA-2-HPMA & 3.52 \\
BC HEA-HEMA & 2.97 \\
BC HEA-NAM & 8.55 \\
BC HEA-NAM-HEA & 13.5 \\
BC HEA-mPEG300 & 10.5 \\
BC HEA-mPEG500 & 8.73 \\
BC HEA-PTMAEMA & 8.78 \\
BC HEA-nBA & 8.93 \\
BC HEA-MA & 9.22 \\
BC HEA-MMA & 9.22 \\
BC NIPAM-2-HPMA & 3.99 \\
BC NIPAM-PTMAEMA & 7.27 \\
BC NIPAM-nBA & 7.51 \\
BC NIPAM-MA & 8.67 \\
BC NIPAM-MMA & 8.84 \\
BC 2-HPMA-nBA & 5.24 \\
BC 2-HPMA-MA & 6.69 \\
BC HEA-MMA & 9.22 \\
BC 2-HPMA-MMA & 6.69 \\
BC HEMA-nBA & 6.40 \\
BC HEMA-MA & 6.92 \\
BC HEMA-MMA & 6.20 \\
\hline
\end{tabular}


Table S6. Characterization data for all homopolymers and homopolymers with PEG conjugated. $\mathrm{M}_{\mathrm{w}}$ and $Ð$ was determined by SEC-MALS, $\mathrm{R}_{\mathrm{g}}$ by SAXS, and $\mathrm{R}_{\mathrm{h}}$ by DLS. P refers to samples that precipitated.

\begin{tabular}{|c|c|c|c|c|c|c|c|c|c|c|c|}
\hline Polymer & $\log P$ & $\begin{array}{l}M_{\text {Theoretical }} \\
\text { (Da) }\end{array}$ & $\begin{array}{c}\mathrm{MW}_{\mathrm{GPC}} \\
\text { (Da) }\end{array}$ & $\begin{array}{c}\text { MW }_{\text {MALS }} \\
\text { (Da) }\end{array}$ & $\oplus_{\mathrm{GPC}}$ & $\oplus_{\text {MALS }}$ & $\begin{array}{c}R_{g} \\
(n m)\end{array}$ & $\begin{array}{c}R_{\mathrm{h}} \\
(\mathrm{nm})\end{array}$ & $R_{g} / R_{h}$ & $\begin{array}{l}\text { Porod } \\
\text { Exponent }\end{array}$ & v \\
\hline HEA & 0.57 & 46,480 & 65,406 & 40,850 & 1.281 & 1.202 & 21.24 & 21.30 & 0.997 & 1.1 & 0.56 \\
\hline HEA $10 \%$ PEG & 0.071 & 93,102 & - & - & - & - & 18.14 & 16.61 & 1.09 & 1.6 & 0.45 \\
\hline NAM & -0.13 & 56,468 & 25,882 & 30,580 & 1.118 & 1.058 & 13.04 & 9.65 & 1.35 & 1.5 & 0.47 \\
\hline NAM $10 \%$ PEG & -0.45 & 94,018 & -- & - & - & - & 12.26 & 9.57 & 1.28 & 1.9 & 0.41 \\
\hline NIPAM & 0.72 & 45,264 & 25,420 & 35,330 & 1.124 & 1.119 & $\mathrm{P}$ & - & - & - & - \\
\hline NIPAM $10 \%$ PEG & 0.18 & 80,248 & -- & - & - & - & $P$ & - & - & - & - \\
\hline DMA & 0.21 & 39,652 & 25,879 & 61,110 & 1.126 & 1.076 & $P$ & - & -- & -- & - \\
\hline DMA 10\% PEG & -0.20 & 94,242 & -- & -- & - & - & 10.53 & 9.06 & 1.16 & 1.5 & 0.47 \\
\hline 2-HPMA & 1.26 & 57,668 & 17,100 & 41,510 & 1.100 & 1.187 & $\mathrm{P}$ & - & - & - & - \\
\hline 2-HPMA 10\% PEG & 0.59 & 69,469 & -- & - & - & - & 17.14 & 14.64 & 1.17 & 1.1 & 0.56 \\
\hline HEMA & 0.85 & 52,056 & 16,601 & 30,220 & 1.093 & 1.205 & $\mathrm{P}$ & - & - & -- & - \\
\hline HEMA $10 \%$ PEG & 0.28 & 67,430 & - & -- & - & - & 20.57 & 20.76 & 0.991 & 1.5 & 0.47 \\
\hline mPEG acrylate & 0.33 & 52,056 & 20,097 & 27,140 & 1.141 & 1.058 & 15.91 & 12.12 & 1.31 & 1.5 & 0.47 \\
\hline mPEG acrylate $10 \%$ PEG & -0.11 & 76,042 & - & -- & - & - & 19.26 & 14.45 & 1.33 & 1.7 & 0.44 \\
\hline
\end{tabular}

Table S7. Characterization data for all random heteropolymers (RH) and block copolymers (BC) only containing various neutral components. All polymerizations were done using a feed ratio of 1:1 for 2component polymers and 1:1:1 for the 3-component polymer (HEA-NAM-HEA). $\mathbf{M}_{\mathrm{w}}$ and $Ð$ was determined by SEC-MALS, $\mathrm{R}_{\mathrm{g}}$ by SAXS, and $\mathrm{R}_{\mathrm{h}}$ by DLS. P refers to precipitated polymers.

\begin{tabular}{|c|c|c|c|c|c|c|c|c|c|c|c|}
\hline Polymer & $\log P$ & $\begin{array}{l}\text { MW }_{\text {Theoretical }} \\
(\mathrm{Da})\end{array}$ & $\begin{array}{c}\mathrm{MW}_{\mathrm{GPC}} \\
(\mathrm{Da})\end{array}$ & $\begin{array}{c}\text { MW }_{\text {MALS }} \\
(\mathrm{Da})\end{array}$ & $\boldsymbol{D}_{\mathrm{GPC}}$ & $\oplus_{\text {MALS }}$ & $\begin{array}{c}R_{g} \\
(n m)\end{array}$ & $\begin{array}{c}R_{\mathrm{h}} \\
(\mathrm{nm})\end{array}$ & $R_{g} / R_{h}$ & $\begin{array}{l}\text { Porod } \\
\text { Exponent }\end{array}$ & $\mathbf{v}$ \\
\hline RH HEA-NIPAM & 0.65 & 45,872 & 29,590 & 23,450 & 1.211 & 1.087 & $\mathrm{P}$ & - & - & - & - \\
\hline RH HEA-NIPAM $10 \%$ PEG & 0.13 & 139,102 & - & - & - & - & 13.22 & 9.61 & 1.38 & 1.4 & 0.49 \\
\hline BC HEA-NIPAM & 0.65 & 45,872 & 27,961 & 25,040 & 1.294 & 1.126 & 18.07 & 18.11 & 0.998 & 1.5 & 0.47 \\
\hline BC HEA-NIPAM $10 \%$ PEG & 0.13 & 139,102 & - & - & - & - & 5.71 & 5.43 & 1.05 & 2.0 & 0.40 \\
\hline RH HEA-2-HPMA & 0.92 & 52,074 & 70,165 & 66,960 & 1.479 & 1.454 & 20.23 & 14.85 & 1.36 & 1.9 & 0.41 \\
\hline RH HEA-2-HPMA 10\% PEG & 0.33 & 89,803 & - & - & - & - & 13.17 & 13.18 & 0.999 & 2.0 & 0.40 \\
\hline BC HEA-2-HPMA & 0.92 & 52,074 & 86,765 & 52,830 & 1.539 & 1.135 & 14.57 & 11.91 & 1.22 & 1.6 & 0.45 \\
\hline BC HEA-2-HPMA $10 \%$ PEG & 0.33 & 84,673 & - & - & - & - & 6.40 & 5.51 & 1.16 & 1.8 & 0.42 \\
\hline RH HEA-NAM & 0.22 & 51,474 & 42,356 & 39,890 & 1.315 & 1.103 & $\mathrm{P}$ & -- & - & - & - \\
\hline RH HEA-NAM $10 \%$ PEG & -0.20 & 144,144 & - & - & - & - & 8.75 & 6.11 & 1.43 & 1.5 & 0.47 \\
\hline BC HEA-NAM & 0.22 & 51,474 & 28,355 & 38,890 & 1.317 & 1.159 & 8.04 & 5.59 & 1.44 & 1.5 & 0.47 \\
\hline BC HEA-NAM $10 \%$ PEG & -0.20 & 130,707 & - & - & - & - & 7.31 & 6.00 & 1.22 & 2.1 & 0.39 \\
\hline RH HEA-NAM-HEA & 0.34 & 49,776 & 32,209 & 15,490 & 1.291 & 1.042 & 7.48 & 6.00 & 1.25 & 1.5 & 0.47 \\
\hline RH HEA-NAM-HEA $10 \%$ PEG & -0.10 & 142,616 & - & - & - & - & 7.47 & 5.66 & 1.32 & 1.5 & 0.47 \\
\hline BC HEA-NAM-HEA & 0.34 & 49,776 & 20,814 & 69,620 & 1.225 & 1.100 & 8.41 & 6.55 & 1.28 & 1.8 & 0.42 \\
\hline BC HEA-NAM-HEA $10 \%$ PEG & -0.10 & 142,616 & - & - & - & - & 10.57 & 5.89 & 1.79 & 1.9 & 0.41 \\
\hline RH NIPAM-2-HPMA & 0.99 & 51,466 & 47,979 & 38,150 & 1.437 & 1.349 & $\mathrm{P}$ & - & - & - & - \\
\hline RH NIPAM-2-HPMA $10 \%$ PEG & 0.39 & 102,426 & - & - & - & - & 12.66 & 9.53 & 1.33 & 2.6 & 0.35 \\
\hline BC NIPAM-2-HPMA & 0.99 & 51,466 & 37,019 & 40,900 & 1.256 & 1.075 & $\mathrm{P}$ & - & - & - & - \\
\hline BC NIPAM-2-HPMA $10 \%$ PEG & 0.39 & 88,442 & - & -- & - & - & 5.65 & 5.01 & 1.13 & 2.1 & 0.39 \\
\hline
\end{tabular}


Table S8. Characterization data for all random heteropolymers (RH) and block copolymers (BC) only containing HEA along with a hydrophobic or hydrophilic monomer. All combinations were done using a feed ratio of HEA:hydrophobic/hydrophilic monomer of 9:1. $\mathrm{M}_{\mathrm{w}}$ and $\varnothing$ was determined by SECMALS, $R_{g}$ by SAXS, and $R_{h}$ by DLS. P refers to precipitated polymers.

\begin{tabular}{|c|c|c|c|c|c|c|c|c|c|c|c|}
\hline Polymer & $\log P$ & $\begin{array}{l}M W_{\text {Theoretical }} \\
\text { (Da) }\end{array}$ & $\begin{array}{c}\mathrm{MW}_{\mathrm{GPC}} \\
(\mathrm{Da})\end{array}$ & $\begin{array}{c}M_{\text {MALS }} \\
\text { (Da) }\end{array}$ & $\oplus_{\mathrm{GPC}}$ & $\boldsymbol{\theta}_{\text {MALS }}$ & $\begin{array}{c}R_{g} \\
(n m)\end{array}$ & $\begin{array}{c}R_{h} \\
(n m)\end{array}$ & $R_{g} / R_{h}$ & $\begin{array}{l}\text { Porod } \\
\text { Exponent }\end{array}$ & $\mathbf{v}$ \\
\hline RH HEA-mPEG300 & 0.59 & 53,832 & 57,328 & 25,720 & 1.387 & 1.050 & 7.14 & 6.27 & 1.14 & 1.7 & 0.44 \\
\hline RH HEA-mPEG300 10\% PEG & 0.086 & 146,266 & -- & -- & - & - & 9.15 & 7.36 & 1.24 & 1.9 & 0.41 \\
\hline BC HEA-mPEG300 & 0.59 & 53,832 & 52,036 & 35,930 & 1.360 & 1.219 & 9.23 & 7.85 & 1.18 & 1.7 & 0.44 \\
\hline BC HEA-mPEG300 10\% PEG & 0.086 & 146,266 & -- & -- & -- & -- & 8.59 & 7.25 & 1.18 & 1.8 & 0.42 \\
\hline RH HEA-mPEG500 & 0.52 & 61,832 & 52,407 & 33,450 & 1.359 & 1.135 & 8.19 & 6.66 & 1.23 & 1.5 & 0.47 \\
\hline RH HEA-mPEG500 10\% PEG & 0.033 & 153,466 & - & -- & -- & -- & 9.57 & 6.61 & 1.45 & 1.9 & 0.41 \\
\hline BC HEA-mPEG500 & 0.52 & 61,832 & 53,839 & 69,690 & 1.442 & 1.011 & 7.39 & 6.85 & 1.08 & 1.6 & 0.45 \\
\hline BC HEA-mPEG500 10\% PEG & 0.033 & 141,828 & - & -- & -- & -- & 10.28 & 10.24 & 1.00 & 1.9 & 0.41 \\
\hline RH HEA-PTMAEMA & -0.18 & 50,140 & 36,566 & 42,020 & 1.279 & 1.099 & 15.03 & 12.36 & 1.22 & 1.5 & 0.47 \\
\hline RH HEA-PTMAEMA $10 \%$ PEG & -0.50 & 93,850 & -- & -- & -- & -- & 15.82 & 12.59 & 1.26 & 1.2 & 0.53 \\
\hline BC HEA-PTMAEMA & -0.18 & 50,140 & 35,137 & 30,770 & 1.367 & 1.159 & 8.97 & 5.92 & 1.52 & 1.7 & 0.44 \\
\hline BC HEA-PTMAEMA $10 \%$ PEG & -0.50 & 131,621 & -- & -- & -- & -- & 10.61 & 6.88 & 1.54 & 1.9 & 0.41 \\
\hline RH HEA-nBA & 0.77 & 46,959 & 46,965 & 30,350 & 1.280 & 1.099 & 5.43 & 4.65 & 1.17 & 1.7 & 0.44 \\
\hline RH HEA-nBA 10\% PEG & 0.22 & 140,080 & -- & -- & -- & -- & 8.32 & 7.24 & 1.15 & 2.0 & 0.40 \\
\hline BC HEA-nBA & 0.77 & 46,959 & 53,521 & 33,030 & 1.497 & 1.169 & 14.93 & 17.60 & 0.848 & 1.6 & 0.45 \\
\hline BC HEA-nBA $10 \%$ PEG & 0.22 & 130,116 & - & - & & - & 13.00 & 13.08 & 0.994 & 1.9 & 0.41 \\
\hline RH HEA-MA & 0.56 & 45,276 & 41,882 & 20,770 & 1.206 & 1.110 & 7.70 & 6.57 & 1.17 & 1.7 & 0.44 \\
\hline RH HEA-MA $10 \%$ PEG & 0.063 & 138,566 & -- & -- & -- & -- & 9.59 & 7.31 & 1.31 & 2.2 & 0.38 \\
\hline BC HEA-MA & 0.56 & 45,276 & 47,715 & 38,550 & 1.388 & 1.149 & 12.34 & 8.82 & 1.40 & 1.6 & 0.45 \\
\hline BC HEA-MA $10 \%$ PEG & 0.063 & 131,289 & - & -- & -- & -- & 10.11 & 9.17 & 1.10 & 2.0 & 0.40 \\
\hline RH HEA-MMA & 0.67 & 45,837 & 43,041 & 25,680 & 1.296 & 1.102 & 14.28 & 9.00 & 1.59 & 1.6 & 0.45 \\
\hline RH HEA-MMA 10\% PEG & 0.15 & 139,071 & -- & -- & -- & -- & 9.32 & 8.75 & 1.07 & 2.7 & 0.35 \\
\hline BC HEA-MMA & 0.67 & 45,837 & 61,382 & 31,030 & 1.419 & 1.181 & 11.56 & 9.35 & 1.24 & 1.7 & 0.44 \\
\hline BC HEA-MMA $10 \%$ PEG & 0.15 & 131,798 & -- & -- & -- & -- & 8.43 & 8.99 & 0.938 & 1.8 & 0.42 \\
\hline
\end{tabular}

Table S9. Characterization data for all random heteropolymers (RH) and block copolymers (BC) only containing NIPAM along with a hydrophobic or hydrophilic monomer. All combinations were done using a feed ratio of NIPAM:hydrophobic/hydrophilic monomer of 9:1. $\mathbf{M}_{\mathrm{w}}$ and $\oslash$ was determined by SEC-MALS, $\mathrm{R}_{\mathrm{g}}$ by SAXS, and $\mathrm{R}_{\mathrm{h}}$ by DLS. P refers to precipitated polymer.

\begin{tabular}{|c|c|c|c|c|c|c|c|c|c|c|c|}
\hline Polymer & $\log P$ & $\begin{array}{l}\mathrm{MW}_{\text {Theoretical }} \\
\text { (Da) }\end{array}$ & $\begin{array}{c}\mathrm{MW}_{\mathrm{GPC}} \\
(\mathrm{Da})\end{array}$ & $\begin{array}{c}\text { MW }_{\text {MALS }} \\
\text { (Da) }\end{array}$ & $\boldsymbol{D}_{\mathrm{GPC}}$ & $\boldsymbol{D}_{\text {MALS }}$ & $\begin{array}{c}R_{g} \\
(n m)\end{array}$ & $\begin{array}{c}R_{h} \\
(n m)\end{array}$ & $\mathbf{R}_{\mathrm{g}} / \mathbf{R}_{\mathrm{h}}$ & $\begin{array}{l}\text { Porod } \\
\text { Exponent }\end{array}$ & $\mathbf{v}$ \\
\hline RH NIPAM-PTMAEMA & -0.042 & 49,046 & 41,395 & 36,480 & 1.403 & 1.043 & 23.47 & 25.40 & 0.92 & 1.5 & 0.47 \\
\hline RH NIPAM-PTMAEMA $10 \%$ PEG & -0.39 & 99,869 & - & - & - & - & 12.38 & 9.22 & 1.34 & 1.9 & 0.41 \\
\hline BC NIPAM-PTMAEMA & -0.042 & 49,046 & 36,449 & 35,850 & 1.355 & 1.073 & 14.99 & 12.56 & 1.19 & 2.9 & 0.34 \\
\hline BC NIPAM-PTMAEMA $10 \%$ PEG & -0.39 & 116,612 & - & - & - & - & 11.57 & 9.32 & 1.24 & 2.0 & 0.40 \\
\hline RH NIPAM-nBA & 0.90 & 45,864 & 47,040 & 18,350 & 1.707 & 1.021 & 6.26 & 5.87 & 1.07 & 1.1 & 0.56 \\
\hline RH NIPAM-nBA $10 \%$ PEG & 0.32 & 125,856 & - & - & - & - & 8.33 & 8.74 & 0.95 & 2.5 & 0.36 \\
\hline BC NIPAM-nBA & 0.90 & 45,864 & 23,061 & 30,250 & 1.181 & 1.123 & 16.57 & 11.44 & 1.45 & 1.9 & 0.41 \\
\hline BC NIPAM-nBA $10 \%$ PEG & 0.32 & 115,834 & - & - & - & - & 14.06 & 14.18 & 0.992 & 2.2 & 0.38 \\
\hline RH NIPAM-MA & 0.70 & 44,181 & 35,710 & 37,550 & 1.339 & 1.085 & $P$ & - & - & - & - \\
\hline RH NIPAM-MA $10 \%$ PEG & 0.17 & 137,580 & - & - & - & - & 8.85 & 8.76 & 1.01 & 1.9 & 0.41 \\
\hline BC NIPAM-MA & 0.70 & 44,181 & 23,481 & 23,730 & 1.237 & 1.061 & $P$ & - & - & - & - \\
\hline BC NIPAM-MA $10 \%$ PEG & 0.17 & 125,139 & - & - & - & - & 8.69 & 7.86 & 1.11 & 1.8 & 0.42 \\
\hline RH NIPAM-MMA & 0.81 & 44,742 & 15,474 & 16,070 & 1.095 & 1.025 & 14.59 & 11.35 & 1.29 & 2.2 & 0.38 \\
\hline RH NIPAM-MMA $10 \%$ PEG & 0.25 & 134,071 & - & - & - & - & 11.71 & 11.08 & 1.06 & 1.9 & 0.41 \\
\hline BC NIPAM-MMA & 0.81 & 44,742 & 20,953 & 21,640 & 1.165 & 1.052 & 16.02 & 19.25 & 0.832 & 2.9 & 0.34 \\
\hline BC NIPAM-MMA $10 \%$ PEG & 0.25 & 127,285 & & -- & -- & -- & 10.77 & 10.92 & 0.986 & 1.8 & 0.42 \\
\hline
\end{tabular}


Table S10. Characterization data for all random heteropolymers $(\mathrm{RH})$ and block copolymers $(\mathrm{BC})$ only containing 2-HPMA along with a hydrophobic monomer. All combinations were done using a feed ratio of 2-HPMA:hydrophobic monomer of $9: 1 . \mathrm{M}_{\mathrm{w}}$ and $Ð$ was determined by SEC-MALS, $\mathrm{R}_{\mathrm{g}}$ by SAXS, and $\mathrm{R}_{\mathrm{h}}$ by DLS. P refers to precipitated polymer.

\begin{tabular}{|c|c|c|c|c|c|c|c|c|c|c|c|}
\hline Polymer & $\log P$ & $\begin{array}{l}\mathrm{MW}_{\text {Theoretical }} \\
\text { (Da) }\end{array}$ & $\begin{array}{c}\mathrm{MW}_{\mathrm{GPC}} \\
(\mathrm{Da})\end{array}$ & $\begin{array}{c}\text { MW }_{\text {MALS }} \\
\text { (Da) }\end{array}$ & $\oplus_{\mathrm{GPC}}$ & $\oplus_{\text {MALS }}$ & $\begin{array}{c}R_{g} \\
(n m)\end{array}$ & $\begin{array}{c}R_{\mathrm{h}} \\
(\mathrm{nm})\end{array}$ & $\mathbf{R}_{\mathrm{g}} / \mathbf{R}_{\mathrm{h}}$ & $\begin{array}{l}\text { Porod } \\
\text { Exponent }\end{array}$ & $\mathbf{v}$ \\
\hline RH 2-HPMA-MMA & 1.29 & 55,906 & 36,953 & 33,370 & 1.438 & 1.158 & $\mathrm{P}$ & - & - & - & - \\
\hline RH 2-HPMA-MMA $10 \%$ PEG & 0.61 & 148,133 & - & - & - & - & 6.95 & 7.62 & 0.912 & 2.9 & 0.34 \\
\hline BC 2-HPMA-MMA & 1.29 & 55,906 & 37,162 & 35,580 & 1.314 & 1.164 & $\mathrm{P}$ & - & - & -- & - \\
\hline BC 2-HPMA-MMA 10\% PEG & 0.61 & 117,615 & - & - & - & -- & 10.00 & 9.74 & 1.03 & 2.0 & 0.40 \\
\hline RH 2-HPMA-nBA & 1.39 & 57,028 & 34,415 & 28,290 & 1.218 & 1.136 & $\mathrm{P}$ & - & - & -- & - \\
\hline RH 2-HPMA-nBA 10\% PEG & 0.69 & 144,914 & 113,371 & 94,090 & 1.709 & 1.215 & 7.64 & 10.17 & 0.751 & 2.9 & 0.34 \\
\hline BC 2-HPMA-nBA & 1.39 & 57,028 & 43,908 & 49,260 & 1.390 & 1.225 & $\mathrm{P}$ & - & - & - & - \\
\hline BC 2-HPMA-nBA 10\% PEG & 0.69 & 105,268 & - & - & - & - & 14.22 & 15.19 & 0.936 & 2.0 & 0.40 \\
\hline RH 2-HPMA-MA & 1.18 & 55,345 & 75,562 & 44,250 & 1.366 & 1.083 & 12.13 & 10.93 & 1.11 & 1.2 & 0.53 \\
\hline RH 2-HPMA-MA $10 \%$ PEG & 0.53 & 83,380 & - & - & - & -- & 17.12 & 21.71 & 0.789 & 2.9 & 0.34 \\
\hline BC 2-HPMA-MA & 1.18 & 55,345 & 47,352 & 44,250 & 1.279 & 1.103 & $\mathrm{P}$ & - & - & - & - \\
\hline BC 2-HPMA-MA $10 \%$ PEG & 0.53 & 117,091 & 112,472 & 94,830 & 1.706 & 1.191 & 8.45 & 10.22 & 0.827 & 2.4 & 0.37 \\
\hline
\end{tabular}

Table S11. Characterization data for all random heteropolymers (RH) and block copolymers (BC) only containing HEMA along with a hydrophobic or hydrophilic monomer. All combinations were done using a feed ratio of HEMA:hydrophobic/hydrophilic monomer of 9:1. $\mathrm{M}_{\mathrm{w}}$ and $\varnothing$ was determined by SEC-MALS, $R_{\mathrm{g}}$ by SAXS, and $\mathrm{R}_{\mathrm{h}}$ by DLS. P refers to precipitated polymer.

\begin{tabular}{|c|c|c|c|c|c|c|c|c|c|c|c|}
\hline Polymer & $\log P$ & $\begin{array}{l}M_{\text {Theoretical }} \\
\text { (Da) }\end{array}$ & $\begin{array}{c}\mathrm{MW}_{\mathrm{GPC}} \\
(\mathrm{Da})\end{array}$ & $\begin{array}{c}\text { MW }_{\text {MALS }} \\
\text { (Da) }\end{array}$ & $\boldsymbol{Ð}_{\mathrm{GPC}}$ & $\boldsymbol{D}_{\text {MALS }}$ & $\begin{array}{c}R_{g} \\
(n m)\end{array}$ & $\begin{array}{c}R_{\mathrm{h}} \\
(\mathrm{nm})\end{array}$ & $R_{g} / R_{h}$ & $\begin{array}{l}\text { Porod } \\
\text { Exponent }\end{array}$ & $\mathbf{v}$ \\
\hline RH HEMA-nBA & 1.02 & 51,977 & 39,726 & 33,250 & 1.719 & 1.295 & $\mathrm{P}$ & - & - & - & - \\
\hline RH HEMA-nBA $10 \%$ PEG & 0.41 & 112,828 & - & - & - & - & 7.00 & 8.52 & 0.822 & 2.6 & 0.35 \\
\hline BC HEMA-nBA & 1.02 & 51,977 & 92,815 & 66,800 & 1.468 & 1.310 & $\mathrm{P}$ & - & - & - & - \\
\hline BC HEMA-nBA $10 \%$ PEG & 0.41 & 111,253 & - & - & - & - & 9.49 & 8.32 & 1.14 & 2.5 & 0.36 \\
\hline RH HEMA-MA & 0.81 & 50,294 & 70,662 & 33,630 & 1.381 & 1.121 & $\mathrm{P}$ & - & - & - & - \\
\hline RH HEMA-MA $10 \%$ PEG & 0.25 & 83,512 & - & - & - & - & 5.34 & 5.76 & 0.927 & 1.8 & 0.42 \\
\hline BC HEMA-MA & 0.81 & 50,294 & 85,878 & 69,320 & 1.619 & 1.308 & $P$ & - & - & - & - \\
\hline BC HEMA-MA $10 \%$ PEG & 0.25 & 114,531 & - & - & - & - & 8.60 & 8.41 & 1.02 & 2.0 & 0.40 \\
\hline RH HEMA-MMA & 0.92 & 50,855 & 21,166 & 18,230 & 1.198 & 1.120 & $\mathrm{P}$ & - & - & - & -- \\
\hline RH HEMA-MMA $10 \%$ PEG & 0.33 & 137,188 & - & - & - & - & 8.21 & 7.71 & 1.06 & 1.9 & 0.41 \\
\hline BC HEMA-MMA & 0.92 & 50,855 & 85,634 & 61,370 & 1.491 & 1.295 & $\mathrm{P}$ & - & - & - & - \\
\hline BC HEMA-MMA $10 \%$ PEG & 0.33 & 108,312 & - & - & - & - & 8.69 & 10.58 & 0.821 & 2.6 & 0.35 \\
\hline RH HEMA-PTMAEMA & 0.08 & 55,158 & 33,289 & 42,210 & 1.414 & 1.124 & 5.33 & 5.29 & 1.01 & 1.5 & 0.47 \\
\hline RH HEMA-PTMAEMA $10 \%$ PEG & -0.30 & 113,123 & - & - & - & - & 11.61 & 8.57 & 1.35 & 1.8 & 0.42 \\
\hline BC HEMA-PTMAEMA & 0.08 & 55,158 & 95,176 & 76,850 & 1.637 & 1.265 & 24.05 & 20.29 & 1.19 & 1.0 & 0.60 \\
\hline BC HEMA-PTMAEMA $10 \%$ PEG & -0.30 & 146,167 & - & - & - & - & 23.89 & 22.23 & 1.07 & 1.4 & 0.49 \\
\hline
\end{tabular}


Table S12. dn/dc values determined for each homopolymer (DP 400) using the Agilent 1200 Series differential RI detector. Weighted averages of $\mathrm{dn} / \mathrm{dc}$ values were used to quantify MALS molar mass of random heteropolymers and block copolymers.

$\begin{array}{ccc}\text { Polymer } & \begin{array}{c}\mathbf{d n} / \mathbf{d c} \\ \mathbf{( m L / g )}\end{array} & \mathbf{R}^{\mathbf{2}} \\ \text { HEA } & 0.152 & 0.9996 \\ \text { NAM } & 0.123 & 0.9913 \\ \text { DMA } & 0.081 & 0.9424 \\ \text { NIPAM } & 0.173 & 0.9981 \\ \text { 2-HPMA } & 0.115 & 0.9992 \\ \text { HEMA } & 0.174 & 1.0000 \\ \text { mPEG acrylate } & 0.123 & 0.9984 \\ \text { nBA } & 0.117 & 0.9861 \\ \text { MA } & 0.081 & 0.9756 \\ \text { MMA } & 0.298 & 0.9961 \\ \text { mPEG300 } & 0.132 & 0.9992 \\ \text { mPEG500 } & 0.146 & 0.9950 \\ \text { PTMAEMA } & 0.078 & 0.9995\end{array}$

a.)

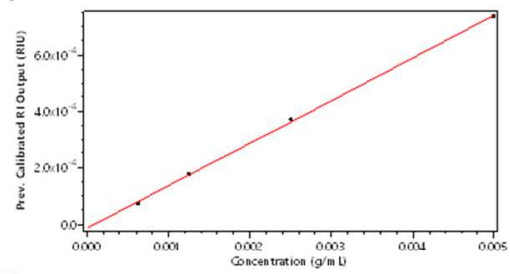

d.)

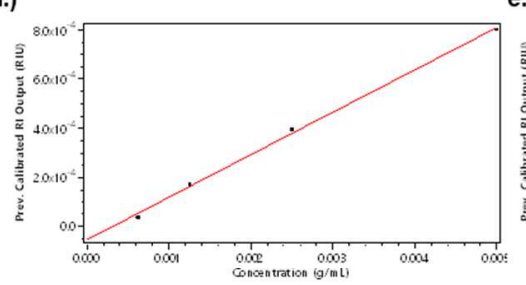

b.)

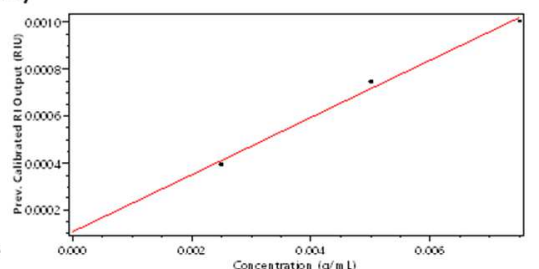

e.)

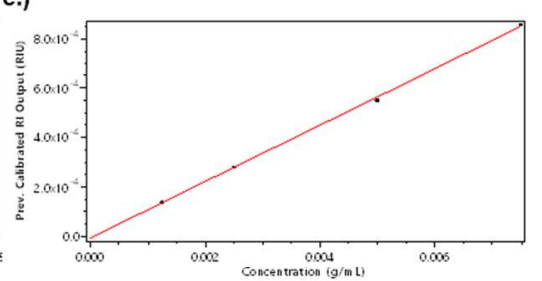

c.)

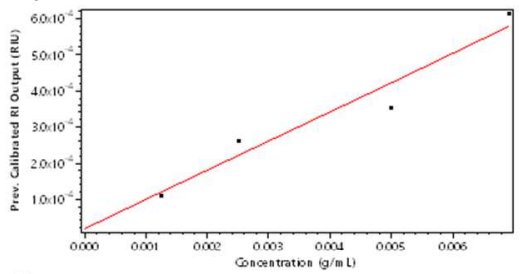

f.)

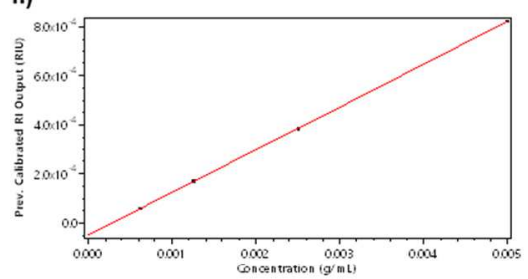

g.)

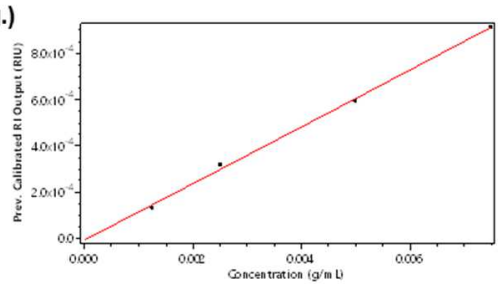

Figure S5. Plots of calibrated RI output (RIU) vs. concentration $(\mathrm{g} / \mathrm{mL})$ obtained from batch injections of the DP 400 neutral homopolymers using the Agilent 1200 Series differential RI detector (plots were processed in Astra chromatography software for light scattering (Wyatt Technology). Displayed are plots for the neutral monomers: (a) HEA; (b) NAM; (c) DMA; (d) NIPAM; (e) 2-HPMA; (f) HEMA; (g) mPEG acrylate. 

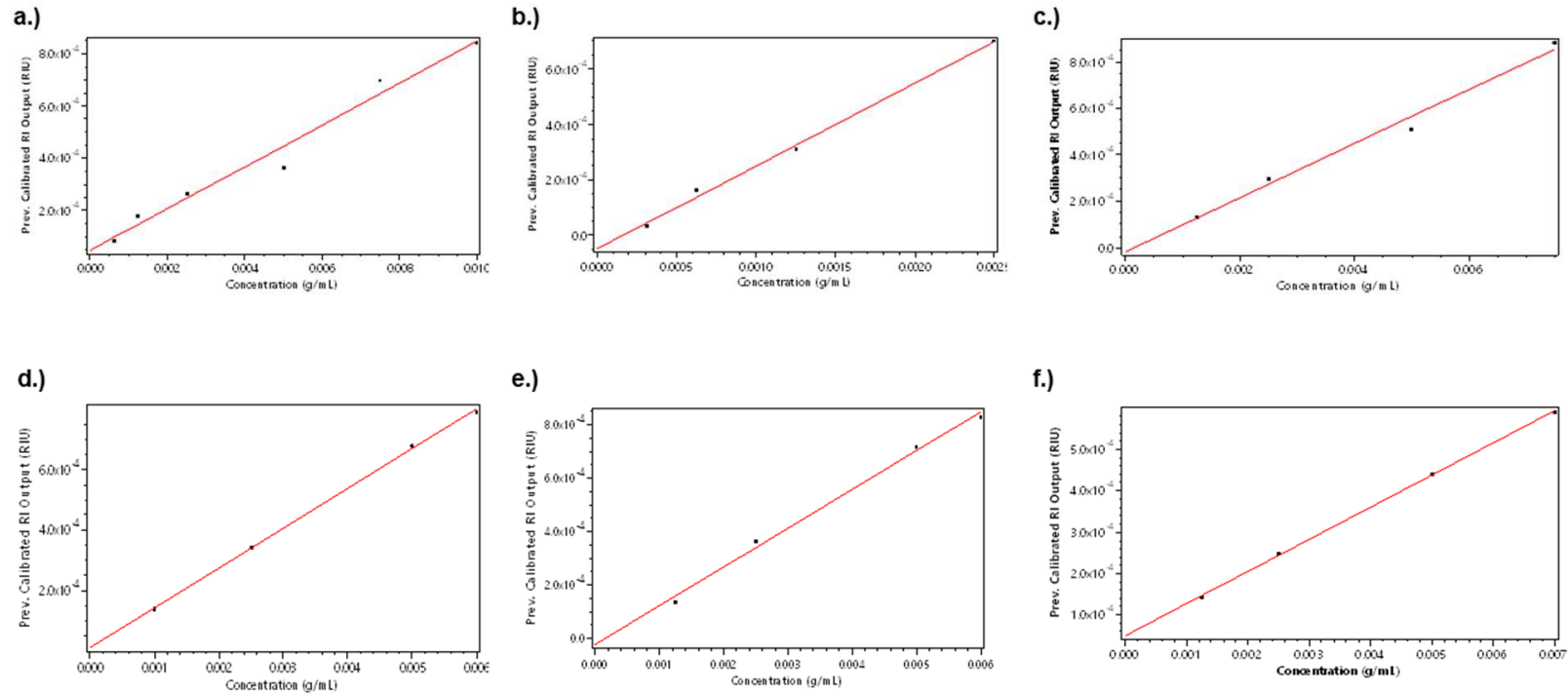

Figure S6. Plots of calibrated RI output (RIU) vs. concentration $(\mathrm{g} / \mathrm{mL})$ obtained from batch injections of the DP 400 hydrophobic and hydrophilic homopolymers using the Agilent 1200 Series differential RI detector (plots were processed in Astra chromatography software for light scattering (Wyatt Technology). Displayed are plots for the neutral monomers: (a) MA; (b) MMA; (c) nBA; (d) mPEG300; (e) mPEG500; (f) PTMAEMA.

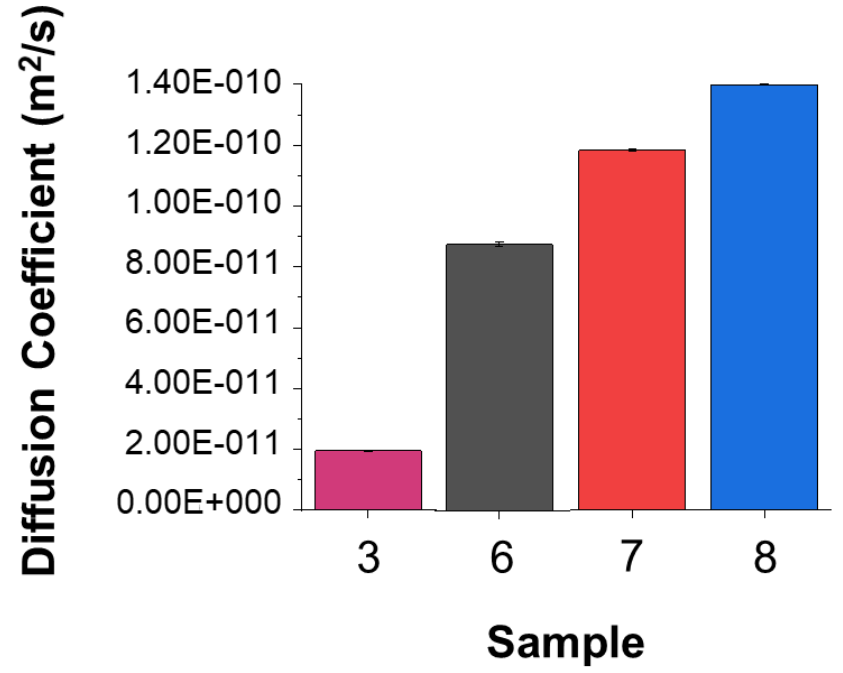

Figure S7. NMR diffusion coefficient summary. Column graph of diffusion coefficients quantified for mPEG acrylate (3), BSA (6), RH 2-HPMA-nBA 10\% PEG (7), and BC 2-HPMA-MA 10\% PEG (8). 


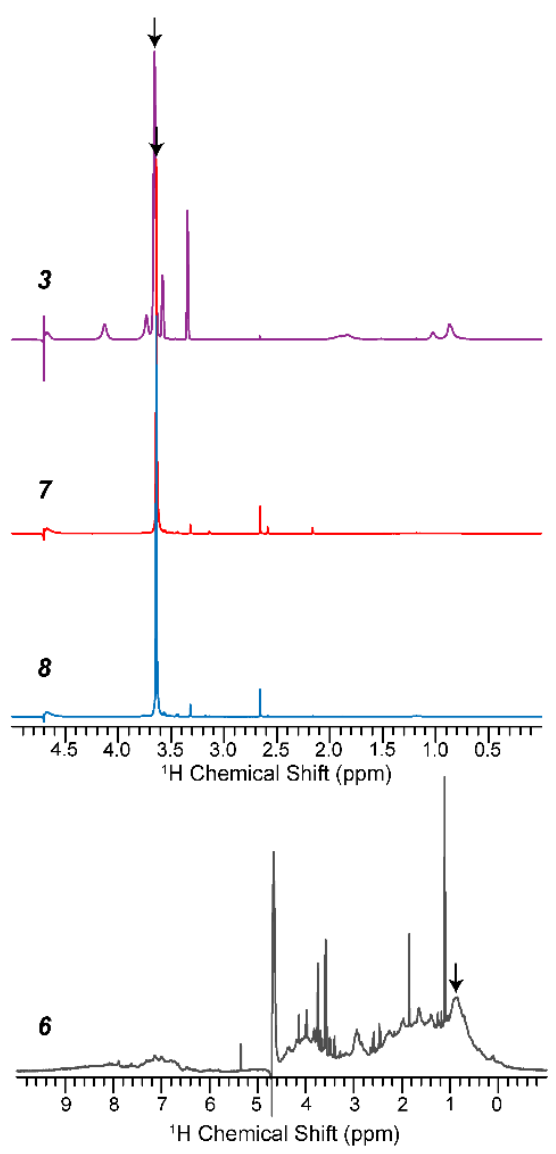

Figure S8. ${ }^{1} \mathrm{H}-\mathrm{NMR}$ (in deuterated PBS) of mPEG acrylate 400 (3), BSA (6), RH 2-HPMA-nBA $10 \%$ PEG (7), BC 2-HPMA-MA 10\% PEG (8). Arrows denote peaks considered for diffusion analysis.
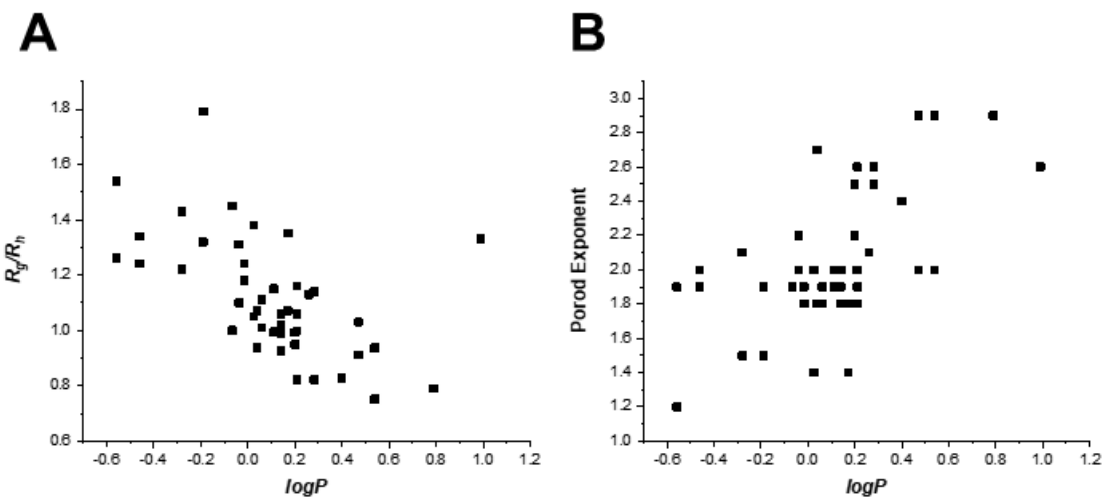

Figure S9. Association between $\log P$ and (A) $R_{g} / R_{h}$ and (B) Porod exponent for all random heteropolymers and block copolymers functionalized with $10 \%$ PEG (combined). Note that in this case $\log P$ refers to $\log P$ of the entire polymer (accounting for the NHS acrylic acid, $\mathrm{DBCO}-\mathrm{NH}_{2}$, and PEG). 

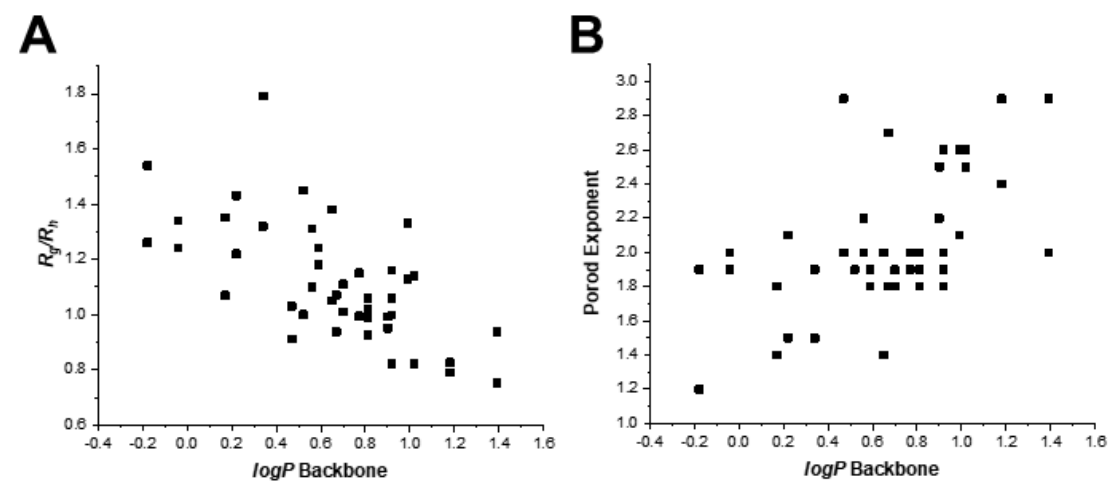

Figure S10. Association between backbone $\log \mathrm{P}$ and $(\mathrm{A}) \mathrm{R}_{\mathrm{g}} / \mathrm{R}_{\mathrm{h}}$ and (B) Porod exponent for all random heteropolymers and block copolymers (pooled) functionalized with $10 \%$ PEG. Note that in this case $\log P$ refers to $\log P$ of the polymer backbone only.

A

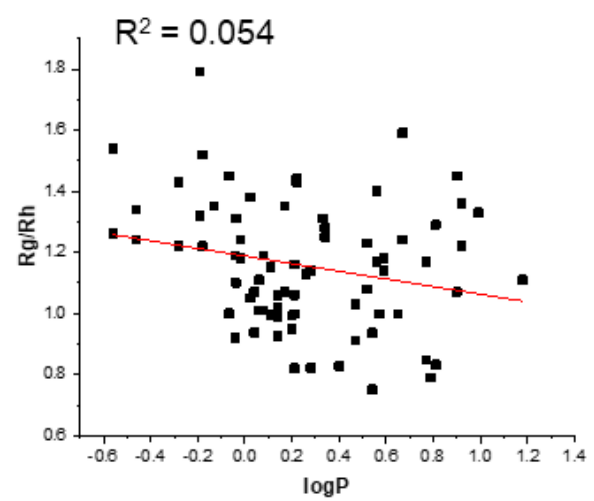

B

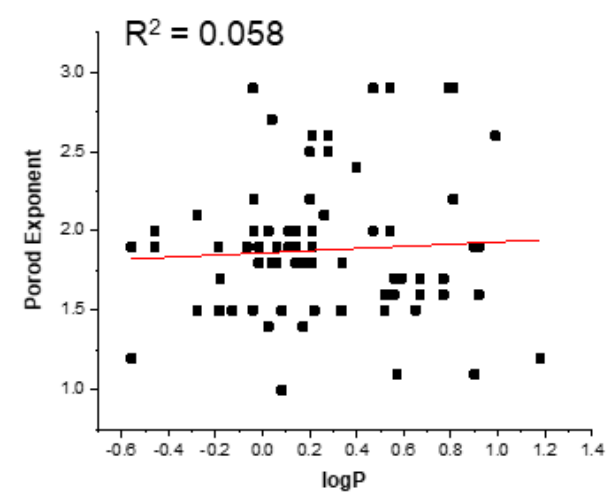

Figure S11. Association between $\log P$ and $(\mathrm{A}) \mathrm{R}_{\mathrm{g}} / \mathrm{R}_{\mathrm{h}}$ and (B) Porod exponent for all polymers characterized. Note that in this case $\log P$ refers to $\log P$ of the entire polymer (accounting for the NHS acrylic acid, $\mathrm{DBCO}-\mathrm{NH}_{2}$, and $\left.\mathrm{PEG}\right)$. The $\mathrm{R}^{2}$ displayed is for a linear fit. 
Table S13. Molecular weight shift (weight-average MW) due to PEG incorporation of RH 2-HPMAnBA 10\% PEG (7) and BC 2-HPMA-MA 10\% PEG (8).

\begin{tabular}{|c|c|c|c|c|}
\hline Polymer & $\begin{array}{c}\mathrm{MW}_{\mathrm{GPC}} \\
(\mathrm{Da})\end{array}$ & $\begin{array}{c}\text { MW }_{\text {MALS }} \\
\text { (Da) }\end{array}$ & $\boldsymbol{D}_{\mathrm{GPC}}$ & $\oplus_{\text {MALS }}$ \\
\hline RH 2-HPMA-nBA 10\% DBCO & 54,827 & 53,430 & 1.466 & 1.068 \\
\hline RH 2-HPMA-nBA 10\% PEG & 113,371 & 94,090 & 1.709 & 1.215 \\
\hline BC 2-HPMA-MA 10\% DBCO & 50,978 & 55,440 & 1.419 & 1.130 \\
\hline BC 2-HPMA-MA 10\% PEG & 112,472 & 94,830 & 1.706 & 1.191 \\
\hline
\end{tabular}

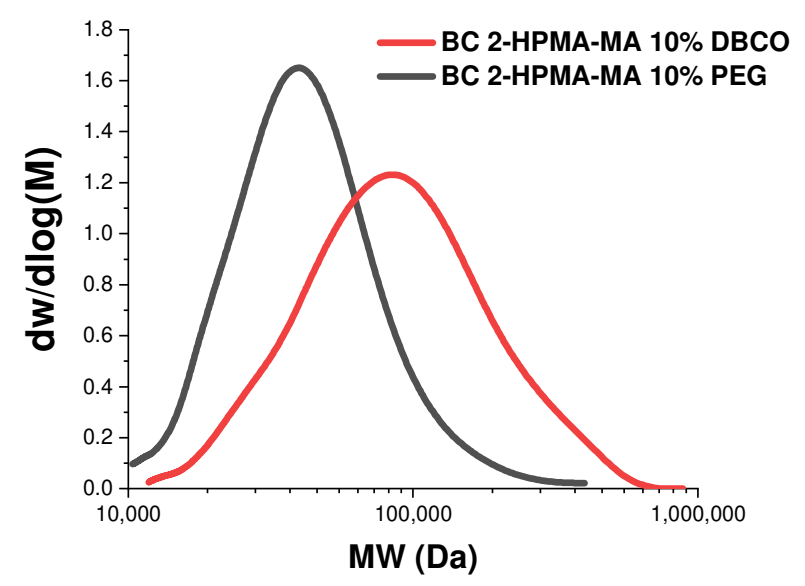

Figure S12. Molecular weight distribution for BC 2-HPMA-MA 10\% DBCO and BC 2-HPMA-MA $10 \%$ PEG in which an increase in molecular weight is due to PEG incorporation. These molecular weight distributions are obtained by RI calibration. 


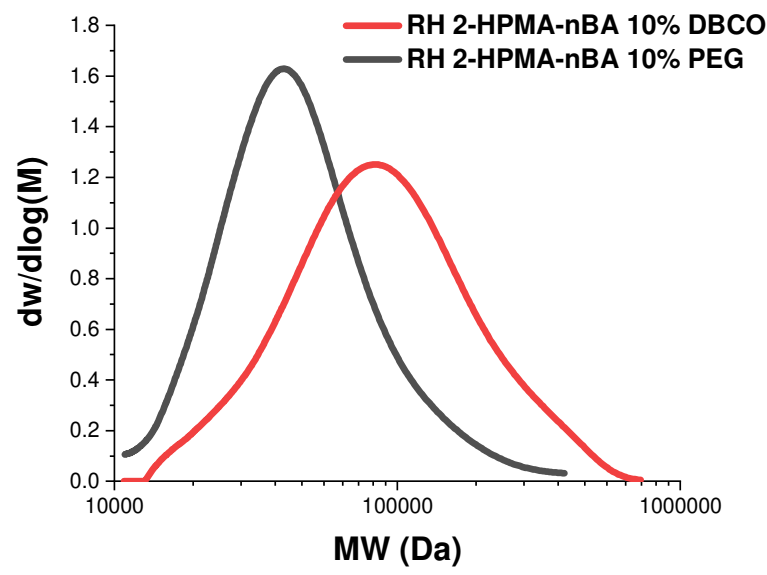

Figure S13. Molecular weight distribution for RH 2-HPMA-nBA 10\% DBCO and RH 2-HPMA-nBA $10 \%$ PEG in which an increase in molecular weight is due to PEG incorporation. These molecular weight distributions are obtained by RI calibration. 